\title{
Cytokines as potential biomarkers of liver toxicity induced by Dioscorea bulbifera L.
}

\author{
Yuchen Sheng ${ }^{2}$, Yibo $\mathrm{Ma}^{1}$, Zhongping Deng ${ }^{2}$, Zhengtao Wang ${ }^{1}$, Lili Ji ${ }^{1, *}$ \\ ${ }^{1}$ The MOE Key Laboratory for Standardization of Chinese Medicines and Shanghai Key Laboratory of Complex Prescription, \\ Institute of Chinese Materia Medica, Shanghai University of Traditional Chinese Medicine, Shanghai, China; \\ ${ }^{2}$ Center for Drug Safety Evaluation and Research, Shanghai University of Traditional Chinese Medicine, Shanghai, China.
}

\begin{abstract}
Summary The present study is designed to search for the serum cytokine biomarker for liver injury induced by Dioscorea bulbifera L., which is a traditionally used herbal medicine in China. Mice were orally given various doses of ethyl acetate extract (EF) isolated from $D$. bulbifera for 12 days. The activity of serum alanine/aspartate transaminases (ALT/AST) was increased in EF (400 mg/kg)-treated mice. Histological assessment further confirmed EF (400 $\mathrm{mg} / \mathrm{kg}$ )-induced liver injury. Results of a cytokine-antibody array demonstrated that there were 10 cytokines up-regulated and 1 cytokine down-regulated in EF (400 mg/kg)-treated mice. Enzyme-linked immunosorbent assay (ELISA) further confirmed the increased level of CD30 ligand (CD30L) and decreased level of interlukin-3 (IL-3) in EF-treated mice. In conclusion, our results demonstrate that the altered expression of $\mathrm{CD30L}$ and IL-3 may be potential biomarkers for hepatotoxicity induced by $D$. bulbifera.
\end{abstract}

Keywords: Dioscorea bulbifera L., hepatotoxicity, cytokines, CD30L, IL-3

\section{Introduction}

Dioscorea bulbifera L. is a native plant in Africa and Asia, and it is also an invasive species in many tropical areas, including Florida in the United States. $D$. bulbifera is generally used to treat thyroid disease (especially goiter) and cancer in clinics in China (1). However, during clinical practice $D$. bulbifera has been found to have hepatotoxicity, which has caused a great obstacle for its application in the clinic (2). Recently, study of the hepatotoxicity of $D$. bulbifera has attracted great interest. Our previous studies have already showed that an ethyl acetate extract (EF) isolated from $D$. bulbifera could induce oxidative stress liver injury $(3,4)$. Meanwhile, our studies and other reports found that diterpenoids were the main hepatotoxic compounds in D. bulbifera, such as diosbulbin B and diosbulbin $\mathrm{D}(3,5)$.

\footnotetext{
*Address correspondence to:

Dr. Lili Ji, The MOE Key Laboratory for Standardization of Chinese Medicines and Shanghai Key Laboratory of Complex Prescription, Institute of Chinese Materia Medica, Shanghai University of Traditional Chinese Medicine, 1200 Cailun Road, Shanghai 201203, China.

E-mail: lichenyue1307@126.com
}

Drug-induced liver injury (DILI) has been a public health issue for many years, and it is the major cause for withdrawal of approved drugs from the market and leads to blockage of development of potential drugs. Generally, herbal medicines are regarded as having no toxicity or side-effects, and they are increasingly used worldwide as dietary supplements $(6,7)$. However, there are increased reports about the hepatotoxicity induced by herbal medicines. For example, herbal medicines as dietary supplements caused about $9 \%$ of cases of DILI, while in Asia the percentage was about $20-55 \%$ cases of DILI $(8,9)$. Thus, liver injury induced by herbal medicines needs to be paid more attention.

Currently, conventional widely used biochemical markers for evaluating liver injury are serum alanine aminotransferase (ALT), aspartate aminotransferase (AST), and cholestatic markers such as alkaline phosphatase (ALP), and $\gamma$-glutamyltransferase $(\gamma \mathrm{GT})$ $(10,11)$. However, all of these biomarkers sometimes can not accurately reflect liver injury (11). Serum ALT activity has also been associated with toxicity of other organs and thus it leads to false positives due to other sources of serum ALT activity because it has specificity beyond the liver (12). Meanwhile, there is a report that serum ALT activity is variable in people, which will also lead to possible false positive or negative results 
(13). Thus, searching for more simple and sensitive biomarkers as a supplementary index for DILI is an urgent problem that needs to be solved.

Cytokines are a diverse group of soluble proteins, peptides or glycoproteins, which have various biological functions. Recently, innate and acquired immunity mediated by cytokines has been reported to play a critical role in DILI, and thus cytokines have the potential value as biomarkers for DILI $(14,15)$. In the present study, we employed a cytokine-antibody array to search for potential cytokine biomarkers for liver injury induced by $D$. bulbifera.

\section{Materials and Methods}

\subsection{Chemicals and reagents}

The rhizomes of D. bulbifera were collected in Qingyang, Anhui Province and authenticated by Prof. Shou-Jin Liu (Anhui College of Traditional Chinese Medicine, Anhui, China). The specimens were deposited in the herbarium of the Institute of Chinese Materia Medica, Shanghai University of Traditional Chinese Medicine. The preparation of ethyl acetate extracts (EF) isolated from D. bulbifera was previously reported in our published papers, and the content of diosbulbin B is $13.72 \%$ (16). The kits for determining ALT/AST activity were obtained from Jiancheng Bioengineering Institute (Nanjing, Jiangsu, China). RayBio ${ }^{\mathrm{TM}}$ Mouse Cytokine Antibody Array III was purchased from RayBiotech, Inc. (Norcross, GA, USA). ELISA kits were purchased from RapidBio (West Hills, CA, USA). Other reagents unless indicated were purchased from Sigma Chemical Co. (St. Louis, MO, USA).

\subsection{Experimental animals}

Specific pathogen free male ICR mice (18-22 g body weight) were purchased from the Chinese Academy of Sciences (Shanghai, China). The mice were fed a standard laboratory diet and given free access to tap water, kept in a controlled room temperature $\left(22 \pm 1{ }^{\circ} \mathrm{C}\right)$, humidity $(65 \pm 5 \%)$, and a 12:12-h light/dark cycle. All mice received humane care in compliance with the institutional animal care guidelines approved by the Experimental Animal Ethical Committee of Shanghai University of Traditional Chinese Medicine.

\subsection{Treatments of animals}

Mice were orally administered EF (100, 200, and 400 $\mathrm{mg} / \mathrm{kg}$, suspended in $0.5 \%$ CMC-Na, $n=7$ ) for 12 days, and $0.5 \%$ CMC-Na was used as a vehicle control $(n=8)$. Animals were killed $24 \mathrm{~h}$ after the last administration. Blood was collected from the eyeball for measurement of ALT, AST, and cytokine analysis. Livers were collected for histological assessment.

\subsection{Serum ALT/AST analysis}

Blood samples obtained from mice of all groups were allowed to coagulate for $2 \mathrm{~h}$ on ice. Serum was then isolated following centrifugation at $840 \times \mathrm{g}$ for $15 \mathrm{~min}$. Serum ALT, and AST activity were measured with kits according to the manufacturer's instructions.

\subsection{Histological examination}

Portions of the liver were fixed in $10 \%$ formalin and embedded in paraffin. Samples were subsequently sectioned $(5 \mu \mathrm{m})$ and stained with hematoxylin-eosin (HE) for further histological analysis.

\subsection{Cytokine antibody array}

Serum cytokines were assayed by Raybio Mouse Cytokine Antibody Array III according to the manufacturer's instructions. This array is capable of simultaneously detecting 62 different cytokines with high specificity. Chemiluminescence signals were visualized by exposure to light sensitive films. Films were digitized, and densitometric quantifications were analyzed with ScanAlyze software, and then the ratio of EF/Control was calculated. Differentially expressed cytokines were defined as over 2 fold alteration between the control and EF-treated groups.

\subsection{ELISA analysis}

Serum was used for ELISA analysis according to the manufacturer's instructions.

\subsection{Statistical analysis}

The results were expressed as Means \pm SEM. SPSS 18.0 was used for statistical analysis. Significance difference between various groups was determined by One-Way ANOVA, and between two groups was evaluated by an independent-sample $t$-test. $p<0.05$ was considered as indicating statistically significant differences.

\section{Results}

\subsection{EF induced liver injury}

As shown in Figure 1A, EF $(400 \mathrm{mg} / \mathrm{kg})$ increased serum ALT and AST activity $(p<0.001)$. Further, histological assessment showed normal liver shape and structure in control mice (Figure 1B). EF (400mg/kg)treated mice exhibited severe liver damage indicated by intrahepatic hemorrhage, lymphocyte infiltration and the destruction of liver structure (Figure 1B).

\subsection{Distinct serum cytokine expression in normal and EF-treated mice}


To identify the cytokine biomarker for D. bulbiferainduced liver injury, we analyzed the content of 62 cytokines in serum by a cytokine antibody array in control and EF (400 mg/kg)-treated mice. Table 1 is the layout of the cytokine antibody array, and Figure 2 is the results of the array. After analyzing, as shown in Table 2 , ten cytokines were up-regulated and one cytokine was down-regulated over 2-fold in EF (400 mg/kg)-treated mice as compared to control. The up-regulated cytokines are $\mathrm{CD} 30 \mathrm{~L}$, fractaline, interferon inducible monokine (CRG2), granulocyte macrophage colony-stimulating factor (GM-CSF), thymus-expressed chemokine (TECK), monocyte chemoattactant protein 5 (MCP-5), IL-4, IL-

A

B
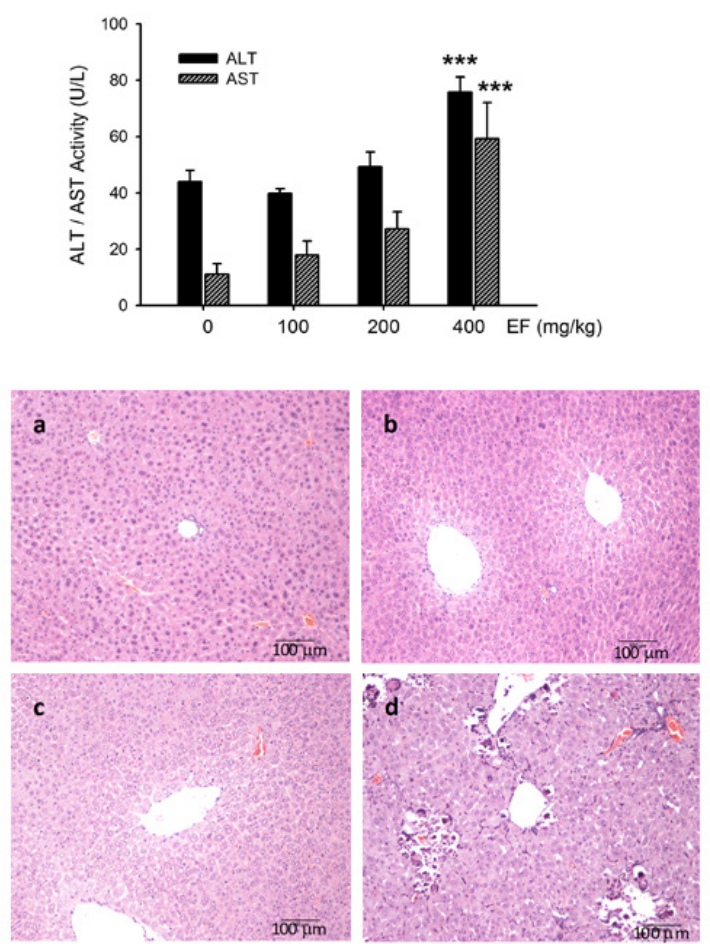

Figure 1. EF induced liver injury. (A) ALT and AST activity. Data are expressed as means \pm SEM $(n=8$ for control and $n=7$ for experimental group). ${ }^{* * *} p<0.001$ compared with control. (B) Histological observation of EFinduced liver injury. a. Control; b. EF (100 mg/kg); c. EF (200 $\mathrm{mg} / \mathrm{kg})$; d. EF (400 mg/kg). Typical images were chosen from each experimental group. (original magnification: $\times 100$ ).

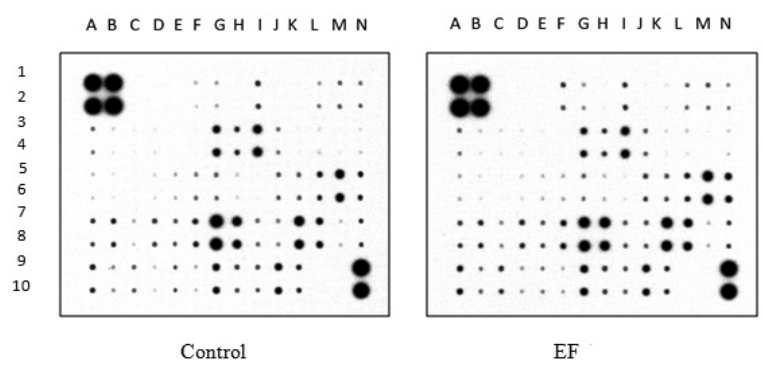

Figure 2. Results of cytokine antibody array. The images of control and EF $(400 \mathrm{mg} / \mathrm{kg})$-treated mice in the cytokine antibody array. $n=8$ for control and $n=7$ for experimental group.

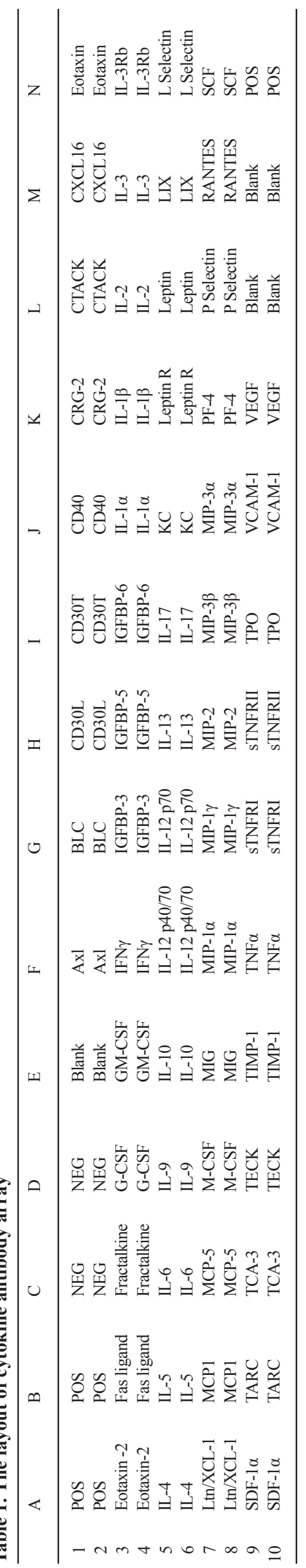


Table 2. List of differentially changed cytokines between control and EF group

\begin{tabular}{lllll}
\hline Row & Column & Cytokine symbol (Used on array) & Full name & The ratio (EF/Cont) \\
\hline 1,2 & F & Axl & Axl receptor tyosine kinase & 3.96 \\
1,2 & H & CD30L & CD30 ligand & 2.22 \\
1,2 & K & CRG-2 & Interferon inducible monokine & 2.17 \\
3,4 & C & Fractalkine & - & 2.20 \\
3,4 & E & GM-CSF & Granulocyte macrophage colony-stimulating factor \\
3,4 & M & IL-3 & Interleukin-3 & 3.42 \\
5,6 & A & IL-4 & Interleukin-4 & 0.26 \\
5,6 & B & IL-5 & Interleukin-5 & 2.39 \\
5,6 & C & IL-6 & Interleukin-6 & 2.02 \\
7,8 & C & MCP-5 & Monocyte chemoattactant protein 5 & 5.63 \\
9,10 & D & TECK & Thymus-expressed chemokine & 2.05 \\
\hline
\end{tabular}

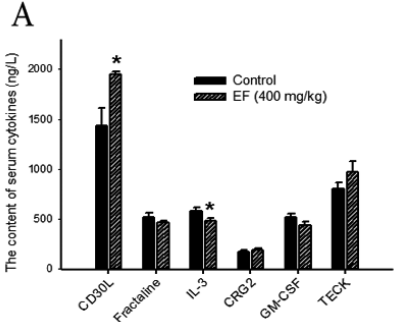

B

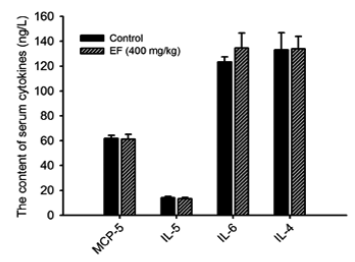

C

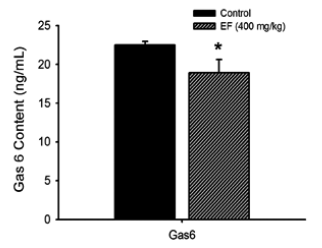

Figure 3. ELISA results. (A) The contents of serum CD30L, fractaline, IL-3, CRG-2, GM-CSF, TECK. (B) The contents of serum MCP-5, IL-5, IL-6, IL-4. (C) The content of serum Gas6. Data are expressed as means \pm SEM ( $n=8$ for control and $\mathrm{n}=7$ for experimental group). ${ }^{*} p<0.05$ compared with control.

5, IL-6, and axl receptor tyosine kinase (Axl), while the down-regulated cytokine is IL-3.

\subsection{ELISA assay of changed cytokine}

Next, ELISA assays were used to validate the change of those cytokines in the cytokine antibody array. Figure $3 \mathrm{~A}$ showed that $\mathrm{CD} 30 \mathrm{~L}$ was increased and IL-3 was decreased in EF $(400 \mathrm{mg} / \mathrm{kg})$-treated mice $(p<0.05)$, which is consistent with the results of the cytokine antibody array. There were no obvious changes of other cytokines such as fractaline, CRG2, GM-CSF, TECK, MCP-5, IL-5, IL-6, or IL-4 (Figures 3A and 3B), which is inconsistent with the results of the cytokine antibody array. Growth arrest-specific 6 (Gas 6), the ligand for axl receptor tyosine kinase (Axl), was decreased in EF $(400 \mathrm{mg} / \mathrm{kg})$-treated mice $(p<0.05)$ in the ELISA assay (Figure 3C).

\section{Discussion}

In China, D. bulbifera is traditionally used to treat thyroid disease. Recently, D. bulbifera has been found to have a good therapeutic effect for some solid cancers, and now is commonly used in cancer therapy in the clinic. However, with the increased application of D. bulbifera in the clinic, its induced hepatotoxicity has widely attracted attention. The present study shows that after treatment with EF isolated from D. bulbifera for 12 days, serum ALT and AST activities were increased. Further, histological assessment demonstrated liver destruction in EF-treated mice. All of these results indicate the potential hepatotoxicity of D. bulbifera, when used for a stage treatment in cancer therapy.

Inflammation is reported to contribute to progressive liver damage induced by exogenous chemical hepatotoxins including drugs like acetaminophen, cycloheximide etc. (17-20). Cytokines are a family of secreted proteins that promote inflammation and participate in both innate and adaptive immune responses which play important roles in DILI $(15,21,22)$. To our knowledge the present study is the first study using a cytokine antibody array to identify potential serum biomarkers for DILI. In the study, the primary screen using cytokine antibody array demonstrated 11 cytokines differentially altered after treatment with EF. Further, ELISA results confirmed that 2 cytokines were significantly changed after treatment with EF, which are CD30L and IL-3. The other 9 cytokines were not changed when measured by ELISA, which may be due to the limitations of the cytokine antibody array. There is already a report demonstrating that a linear response between the chemiluminescent signal and quantity may not always exist, which may lead to the false results shown in the cytokine antibody array (23), so further verification experiments such as ELISA are necessary. 
$\mathrm{CD} 30 \mathrm{~L}$ is the ligand for $\mathrm{CD} 30$, which represents the newest member of the tumor necrosis factor receptor (TNF-R) family (24). Up-regulation of CD30/CD30L is associated with several hematological malignancies such as Hodgkin's disease (HD), anaplastic large cell lymphoma (ALCL) and subsets of Non-Hodgkin's lymphomas (NHL's) (25). Recently, the interaction of CD30/CD30L was reported to play some role in the crosstalk between natural killer and dendritic cells, and is critical for humoral immunity $(26,27)$. Meanwhile, some studies demonstrate that $\mathrm{CD} 30 \mathrm{~L} / \mathrm{CD} 30$ might be useful as a novel biological therapy for allergic rhinitis, inflammatory bowel disease, autoimmune diabetes, and chronic inflammatory skin diseases like psoriasis or atopic dermatitis (28-31). However, there is no report about whether there is some role of CD30L in liver injury. The present study found that serum CD30L was increased in EF-treated mice, which suggests potential involvement of CD30L in D. bulbifera-induced liver injury. This result is the first report about the potential role of $\mathrm{CD} 30 \mathrm{~L}$ in liver injury, and the concrete mechanism needs further investigation.

IL-3 is a key cytokine which promotes survival, proliferation, differentiation and maturity of bone marrow-derived hematopoietic stem cells, and thus it is widely studied to treat different states of bone marrow failure or hematologic malignancies $(32,33)$. In addition, recent studies demonstrate that IL-3 participates in the response of the organism to various types of stress (34). Although there are already reports about the change of IL-3 in hemorrhage or alcoholinduced liver injury, the role of IL-3 in liver injury is still not clear $(35,36)$. Our results demonstrate the down-regulation of serum IL-3 in EF-treated mice, which suggest potential involvement of IL-3 in $D$. bulbifera-induced liver injury.

In conclusion, the present study demonstrates that serum CD30L and IL-3 levels were up-regulated and down-regulated respectively in EF-treated mice, which demonstrates that inflammation may contribute to the liver injury induced by $D$. bulbifera, and the concrete mechanism needs further investigation.

\section{Acknowledgements}

This work was financially supported by the Program of Shanghai Municipal Education Commission (2012JW26); the Program for New Century Excellent Talents in University (NCET-11-1054); and State major science and technology special projects during the 12th five year plan (2012ZX09505001-002).

\section{References}

1. Jiangsu New Medical College. Chinese Dictionary (Second Volume). Shanghai Science and Technology Press, Shanghai, China, 1977; pp. 2059-2061.
2. Liu JR. Two cases of toxic hepatitis caused by Dioscorea bulbifera L. Adverse Drug Reactions J. 2002; 2:129-130.

3. Wang JM, Liang QN, Ji LL, Liu H, Wang CH, Wang ZT. Gender-related difference in liver injury induced by Dioscorea bulbifera L. rhizome in mice. Hum Exp Toxicol. 2011; 30:1333-1341.

4. Wang JM, Liu H, Ji LL, Wang ZT. Study of the hepatotoxicity induced by Dioscorea bulbifera L. rhizome in mice. BioSci Trends. 2010; 4:79-85.

5. Ma M, Jiang Z, Ruan J, Tan X, Liu J, Wang C, Zha XM, Zhang L. The furano norclerodane diterpenoid disobulbin-D induces apoptosis in normal human liver L-02 cells. Exp Toxicol Pathol. 2012; 64:611-618.

6. Su D, Li L. Trends in the use of complementary and alternative medicine in the United States: 2002-2007. J Health Care Poor Underserved. 2011; 22:296-310.

7. Nin Chau T, Cheung WI, Ngan T, Lin J, Lee KW, Tat Poon W, Leung VK, Mak T, Tse ML. Causality assessment of herb-induced liver injury using multidisciplinary approach and Roussel Uclaf Causality Assessment Method (RUCAM). Clin Toxicol (Phila). 2011; 49:34-39.

8. Chalasani N, Fontana RJ, Bonkovsky HL, Watkins PB, Davern T, Serrano J, Yang H, Rochon J. Causes, clinical features, and outcomes from a prospective study of drug-induced liver injury in the United States. Gastroenterology. 2008; 135:1924-1934.

9. Wai CT, Tan BH, Chan CL, Sutedja DS, Lee YM, Khor C, Lim SG. Drug-induced liver injury at an Asian center: A prospective study. Liver Int. 2007; 27:465-474.

10. Kim WR, Flamm SL, Di Bisceglie AM, Bodenheimer HC. Serum activity of alanine aminotransferase (ALT) as an indicator of health and disease. Hepatology. 2008; 47:1363-1370.

11. Cummings J, Ward TH, Greystoke A, Ranson M, Dive C. Biomarker method validation in anticancer drug development. Br J Pharmacol. 2008; 153:646-656.

12. Ozer J, Ratner M, Shaw M, Bailey W, Schomaker S. The current state of serum biomarkers of hepatotoxicity. Toxicology. 2008; 245:194-205.

13. Ruhl CE, Everhart JE. Diurnal variation in serum alanine aminotransferase activity in the US population. J Clin Gastroenterol. 2013; 47:165-173.

14. Chalasani N, Bjornsson E. Risk factors for idiosyncratic drug-induced liver injury. Gastroenterology. 2010; 138:2246-2259.

15. Laverty HG, Antoine DJ, Benson C. The potential of cytokines as safety biomarkers for drug-induced liver injury. Eur J Clin Pharmacol. 2010; 66:961-976.

16. Wang JM, Ji LL, Branford-White C, Wang ZY, Shen KK, Liu H, Wang ZT. Antitumor activity of Dioscorea bulbifera L. rhizome in vivo. Fitoterapia. 2012; 83:388394.

17. Szabo G, Mandrekar P, Dolganiuc A. Innate immune response and hepatic inflammation. Semin Liver Dis. 2007; 27:339-350.

18. Prandota J. Important role of proinflammatory cytokines/other endogenous substances in druginduced hepatotoxicity: Depression of drug metabolism during infections/inflammation states, and genetic polymorphisms of drug-metabolizing enzymes/cytokines may markedly contribute to pathology. Am J Ther. 2005; 12:254-261

19. Jaeschke H, Williams CD, Ramachandran A, Bajt ML. Acetaminophen hepatotoxicity and repair: The role of sterile inflammation and innate immunity. Liver Int. 
2012; 32:8-20.

20. Kumagai K, Kiyosawa, N, Ito K, Yamoto T, Teranishi M, Nakayama H, Manabe S. Influence of kupffer cell inactivation on cycloheximide-induced hepatic injury. Toxicology. 2007; 241:106-118.

21. Holt MP, Ju C. Mecahnsims of drug-induced liver injury. AAPS J. 2006; 8:E48-54.

22. Ju C, Reilly T. Role of immune reactions in drug-induced liver injury (DILI). Drug Metab Rev. 2012; 44:107-115.

23. Budowle B, Hudlow WR, Lee SB, Klevan L. Using a CCD camera imaging system as a recording device to quantify human DNA by slot hybridization. Biotechniques. 2001; 30:680-685.

24. Horie R, Watanabe T. CD30: Expression and function in health and disease. Semin Immunol. 1998; 10:457-470.

25. Oflazoglu E, Grewal IS, Gerber H. Targeting CD30/ $\mathrm{CD} 30 \mathrm{~L}$ in oncology and autoimmune and infammatory diseases. Adv Exp Med Biol. 2009; 647:174-185.

26. Kennedy MK, Willis CR, Armitage RJ. Deciphering CD30 ligand biology and its role in humoral immunity. Immunology. 2006; 118:143-152.

27. Simhadri VL, Hansen HP, Simhadri VR, Reiners KS, Bessler M, Engert A, von Strandmann EP. A novel role for reciprocal CD30-CD30L signaling in the cross-talk between natural killer and dendritic cells. Biol Chem. 2012; 393:101-106.

28. Fuchiwaki T, Sun X, Fujimura K, Yamada H, Shibata K, Muta H, Podack ER, Kawauchi H, Yoshikai Y. The central role of $\mathrm{CD} 30 \mathrm{~L} / \mathrm{CD} 30$ interactions in allergic rhinitis pathogenesis in mice. Eur J Immunol. 2011; 41:2947-2954.

29. Somada S, Muta H, Nakamura K, Sun X, Honda K, Ihara E, Akiho H, Takayanagi R, Yoshikai Y, Podack ER,
Tani K. CD30 ligand/CD30 interaction is involved in pathogenesis of inflammtory bowel disease. Dig Dis Sci. 2012; 57:2031-2037.

30. Chakrabarty S, Nagata M, Yasuda H, Wen L, Nakayama M, Chowdhury SA, Yamada K, Jin Z, Kotani R, Moriyama H, Shimozato O, Yagita H, Yokono K. Critical roles of CD30/CD30L interactions in murine autoimmune diabetes. Clin Exp Immunol. 2003; 133:318-325.

31. Fischer M, Harvima IT, Carvalho RFS, Moller C, Naukkarinen A, Enblad G, Nilsson G. Mast cell CD30 ligand is upregualted in cutaneous inflammation and mediates degranulation-independent chemokine secretion. J Clin Invest. 2006; 116:2748-2756.

32. Eder M, Geissler G, Ganser A. IL-3 in the clinic. Stem cells. 1997; 15:327-333.

33. Johnson DE. Regulation of survival pathways by IL-3 and induction of apoptosis following IL-3 withdrawal. Front Biosci. 1998; 3:d313-324.

34. Bessler H, Bergman M, Salman H. Interleukin-3 and stress. Biomed Pharmacother. 2000; 54:299-304.

35. Anaya-Prado R, Toledo-Pereyra LH, Guo RF, Reuben J, Ward PA, Walsh J. The attentuation of hemorrhageinduced liver injury by exogenous nitric oxide, L-arginine, and inhibition of inducible nitric oxide synthase. J Invest Surg. 2003; 16:247-261.

36. Zhang XG, Xu P, Liu Q, Yu CH, Zhang Y, Chen SH, Li YM. Effect of tea polyphenol on cytokine gene expression in rats with alcoholic liver disease. Hepatobiliary Pancreat Dis Int. 2006; 5:268-272.

(Received December 5, 2013; Revised January 22, 2014; Accepted February 6, 2014) 